\title{
Resumen de los principios de la OCDE para el gobierno corporativo*
}

\author{
Traducción del inglés, Francisco López Herrera \\ y Jorge Ríos Szalay
}

\section{Introducción}

T a integridad de las empresas y de los mercados es un factor fundamental para Lla vitalidad y la estabilidad de nuestras economías. De manera que el buen gobierno corporativo (las reglas y prácticas que gobiernan la relación entre los administradores y los accionistas de las corporaciones, así como con grupos de interés como los empleados y los acreedores) contribuye al crecimiento y estabilidad financiera al apuntalar la confianza del mercado, la integridad del mercado financiero y la eficiencia económica. Los recientes escándalos corporativos han hecho que los gobiernos, los organismos de regulación, las empresas, los inversionistas y el público en general centren su atención sobre las debilidades de los sistemas de gobierno corporativo y la necesidad de enfrentar este asunto.

Los Principios de la OCDE para el Gobierno Corporativo proporcionan guías específicas para que los creadores de políticas, los organismos de regulación y los participantes en el mercado mejoren el marco legal, institucional y regulatorio que sostiene al gobierno corporativo, enfocándose en las empresas cuya propiedad se

\footnotetext{
* Publicado originalmente en inglés y francés por la OCDE con los siguientes títulos:

Policy Brief: The OECD Principles of Corporate Governance, (C) OECD 2004, reservados todos los derechos. Synthèses: Les principes de gouvernement d'entreprise à l'OCDE, (C) OECD 2004, reservados todos los derechos.

La calidad de la traducción al español y su coherencia con el texto original son responsabilidad de la Universidad Nacional Autónoma de México.

Originally published by the OECD in English and French under the titles:

Policy Brief: The OECD Principles of Corporate Governance, (C) OECD 2004, All rights reserved. Synthèses: Les principes de gouvernement d'entreprise à l'OCDE, (C) OECD 2004, All rights reserved. The quality of the Spanish translation and its coherence with the original is the responsibility of Universidad Nacional Autónoma de México.
} 
negocia públicamente. Dichos principios brindan también sugerencias prácticas para los mercados bursátiles, los inversionistas, las corporaciones y otras partes que juegan algún papel en el proceso del desarrollo de un buen gobierno corporativo, por lo que se han incluido dentro de las 12 normas clave para la estabilidad financiera pronunciadas por el Foro de Estabilidad Financiera.

Los Principios de la OCDE se emitieron originalmente en 1999 convirtiéndose desde entonces en la referencia internacional para el gobierno corporativo y la base para diversas iniciativas de reforma tanto gubernamentales como del sector privado. Mediante un proceso de consultas abiertas que se extendieron a países no miembros de la OCDE a través del trabajo de mesas redondas regionales sobre gobierno corporativo, los Principios se revisaron en 2003 para tomar en cuenta los hechos posteriores a 1999. En abril de 2004 los gobiernos de los países miembros de la OCDE acordaron los nuevos Principios y este resumen delinea sus principales características e ilustra cómo abordan los aspectos clave del gobierno corporativo.

\section{¿Qué son los Principios y cuáles aspectos abordan?}

Los Principios cubren seis áreas clave del gobierno corporativo: seguridad para las bases de un marco para un gobierno corporativo eficaz; los derechos de los accionistas; el trato equitativo para los accionistas; el papel de los grupos de interés en el gobierno corporativo; la revelación y la transparencia de la información corporativa relevante; así como las responsabilidades del consejo de administración (véase el cuadro 1). Hay notas explicativas para cada área que indican también el rango de medidas políticas que han demostrado ser exitosas en el logro de sus objetivos. La clave para su éxito es que los Principios sientan bases y no son prescriptivos de forma tal que mantienen su pertinencia para diferentes contextos legales, económicos y sociales. 


\section{Cuadro 1. Las principales áreas de los Principios de la OCDE}

I. Seguridad para las bases de un marco para un gobierno corporativo eficaz El marco para el gobierno corporativo debe promover mercados eficientes y transparentes, ser consistente con las leyes vigentes y articular en forma clara las responsabilidades entre las diferentes autoridades encargadas de la superivisión, la regulación y el cumplimiento de las leyes.

II. Los derechos de los accionistas y las funciones clave de los propietarios El marco para el gobierno corporativo debe proteger los derechos de los accionistas y facilitar su ejercicio.

III. El trato equitativo para los accionistas

El marco para el gobierno corporativo debe asegurar el trato equitativo para todos los accionistas, incluyendo a los minoritarios y extranjeros. Todos los accionistas deben tener la oportunidad de que se les haga justicia eficaz en caso de que se violen sus derechos.

IV. El papel de los grupos de interés en el gobierno corporativo El marco para el gobierno corporativo debe reconocer los derechos de los grupos de interés que se han establecido por la ley o mediante acuerdos mutuos, también debe fomentar la cooperación activa entre las empresas y los grupos de interés para la creación de riqueza, empleo y la sustantibilidad de las empresas financieramente exitosas.

V. Revelación y transparencia de la información corporativa relevante El marco para el gobierno corporativo debe dar seguridad de que se revela oportunamente y con exactitud la información sobre todos los asuntos respecto a la empresa, incluyendo la relacionada con su situación financiera, su desempeño, sus propietarios y su dirección.

VI. Responsabilidades del consejo de administración

El marco para el gobierno corporativo debe dar seguridad para que la empresa sea dirigida estratégicamente, para que exista un control eficaz de la administración por parte del consejo de administración y para que éste rinda cuentas a la empresa y a los accionistas. 
Una parte integral de los Principios son los requisitos básicos del marco institucional y legal-regulatorio que se necesitan para sostener un gobierno corporativo eficaz. El texto incluye principios para el desarrollo de dicho marco y se ocupa de la necesidad de leyes y regulaciones de observancia obligatoria y sostenidas por autoridades con capacidad coactiva. La experiencia alrededor del mundo muestra que aunque en varios países se ha introducido exitosamente el poderoso concepto de la empresa que cotiza en el mercado de valores a menudo el rezago de los sistemas legal y regulatorio ha propiciado casos de abuso en detrimento de los accionistas minoritarios, así como perspectivas de crecimiento reducido cuando los mercados financieros pierden credibilidad o, en primera instancia, fracasan en alcanzarla.

Otras áreas cubiertas por los Principios tienen como intención el establecimiento de un sistema eficaz de restricciones y equilibrios entre los consejos de administración y la administración. Por ejemplo, los administradores profesionales juegan un papel clave en las modernas empresas que cotizan en los mercados de valores o cuya propiedad se encuentra ampliamente diseminada, pero para evitar posibles usos erróneos de su posición requieren, inter alia, de control eficaz por parte del consejo de administración. Los Principios enfatizan que dicha supervisión no debe involucrar sólo la administración cotidiana, sino más bien asegurar la orientación estratégica de la empresa y la vigilancia de los controles internos.

¿Pero quién vigila a los vigilantes? El consejo de administración es a su vez responsable ante los accionistas quienes, de acuerdo con los Principios, deben ser capaces de ejercer sus derechos fundamentales de propiedad, incluyendo el nombramiento y la remoción de los miembros del consejo de administración, y deben recibir un trato equitativo por parte de la empresa. El empleo eficaz de los derechos de propiedad para vigilar e influir en el consejo de administración requiere normas básicas de revelación y transparencia de la información, otra área que también es considerada por estos principios. No obstante, la realidad es a menudo más compleja cuando la empresa y su administración están bajo el control de un accionista dominante, implicando para la vigilancia un caso diferente, pero que también es cubierto por estos principios. Finalmente, para que la empresa sea exitosa se requiere considerar también a los grupos de interés, tales como los de empleados y acreedores que proporcionan recursos a la empresa y quienes necesitan también acceso a información oportuna y pertinente. La atención a los grupos de interés es un rasgo distintivo de los Principios. 
Los Principios de la $O C D E$ son de gran pertinencia para muchos casos recientes de empresas con un alto perfil de quiebra corporativa. Por ejemplo, en varios casos los consejos de administración parecen haber estado adormecidos o incluso se convirtieron en una parte de la administración en lugar de ser vigilantes activos del desempeño de ésta; en otros casos parecen haber actuado sólo como figuras de adorno respondiendo a los deseos de un accionista dominante. Los accionistas parecen haber sido pasivos o ineficaces para sancionar a los consejos de administración y en varios casos los accionistas que mantienen el control han visto por sus intereses a expensas de los accionistas minoritarios. Las complejas instituciones financieras y estructuras corporativas alrededor del mundo también han puesto de manifiesto la cuestión del conflicto de intereses, el cual ha sido más ostensible en algunas investigaciones sobre el corretaje y en la administración de fondos de inversión. Los Principios se han ocupado siempre de estos asuntos, pero la versión revisada les da mayor énfasis.

\section{¿Cómo fortalecer el papel de los accionistas como propietarios?}

A menudo se piensa que el incremento significativo de las tenencias accionarias por parte de los inversionistas institucionales en diversos países durante los años recientes ha llevado a la formación de un grupo grande y poderoso de votantes a favor de leyes para la vigilancia de las empresas. De hecho, los inversionistas institucionales, especialmente aquellos que actúan como fiduciarios en esquemas de inversión colectiva y fondos de pensiones, continúan jugando un papel limitado en el gobierno corporativo al igual que los propietarios de las empresas. El asunto central para la política no es necesariamente si ellos deben tener derechos adicionales al igual que los accionistas, sino más bien que no hacen un uso informado de los derechos que ya tienen. Por supuesto, esto va en detrimento del inversionista que les ha confiado sus fondos. Desde la perspectiva de los Principios de la $O C D E$, la decisión para ejercer los derechos de voto de manera informada está relacionada con los costos y beneficios de votar, por lo que es el incentivo para hacerlo lo que requiere mejorarse en muchos de los casos, en parte por medio de iniciativas políticas. Los Principios no obligan a que los inversionistas institucionales con capacidad para actuar como fiduciarios emitan su voto de acuerdo con su posición accionaria, pero sí los conminan a que revelen sus políticas de voto. Cuando estas políticas incluyen el uso activo de los derechos de propiedad, los Principios les recomiendan también que hagan pública la forma en que las instrumentan, incluyendo los recursos que reservan para este propósito. Dichos Principios tam- 
bién llaman a que se eliminen los impedimentos al voto transfronterizo y a que las empresas eviten el hacer innecesariamente difícil y costoso el ejercicio del voto.

Con respecto al ejercicio de los derechos de propiedad, los Principios aconsejan que los accionistas tengan tanto el derecho a remover a los miembros del consejo de administración como a participar en su nominación. Los accionistas deben tener poder para interrogar al consejo de administración en la asamblea de accionistas y para incluir puntos de su interés en la agenda. Los Principios de la $O C D E$ exhortan a que el consejo de administración formule y haga pública una política de remuneración para sus miembros y ejecutivos clave, resaltando el vínculo entre ella y el desempeño. Los accionistas también deben tener el poder para expresar sus opiniones acerca de esta política y las remuneraciones mediante participaciones en el capital, como las opciones sobre acciones, deben estar sujetas a su aprobación.

El uso informado de los derechos de propiedad es costoso incluso para los inversionistas institucionales, quienes en muchos casos piensan que sus participaciones individuales en una empresa no son suficientemente grandes como para justificar estos costos. Con el fin de superar esta situación, los Principios recomiendan que las autoridades permitan e incluso animen a que dichos inversionistas, y otros accionistas, cooperen y actúen de manera coordinada. Sin embargo, una advertencia importante es que esa cooperación no esté dirigida a la manipulación del mercado o a obtener control de la empresa sin pasar por los procedimientos aceptados para tomarlo. La mejor coordinación en la nominación y elección de los miembros del consejo de administración, el incluir en la agenda los puntos propuestos y el sostener un diálogo directo con la empresa son métodos bien recibidos para mejorar el gobierno corporativo. En general, la posición de los Principios de la $O C D E$ es que los costos de una propiedad eficaz pueden y deben reducirse.

Como parte de su enfoque sobre la participación de los inversionistas institucionales, los Principios de la OCDE llaman a los países a que retiren las barreras regulatorias que obstruyen el diálogo continuo entre los inversionistas y las empresas. Al mismo tiempo, reconociendo que esas relaciones estrechas pueden degenerar en abusos, particularmente cuando existen conflictos de interés inherentes, dichos principios recomiendan que la revelación general de información al mercado siga siendo la norma. Toda información adicional que la empresa entregue a los inversionistas institucionales debe tener como objetivo ayudarles a entender el antecedente de dicha información. 


\section{¿Cómo abordan los Principios los conflictos de intereses?}

Una de las lecciones más sorprendentes de los últimos años es que los conflictos de intereses se encuentran ampliamente diseminados y que a menudo pueden llevar a conductas que van en detrimento de accionistas, inversionistas y otros grupos de interés. Dado que los conflictos de intereses pueden asumir formas diferentes, estos conflictos se tratan en varias secciones diferentes de los Principios. A manera de método general, estos Principios recomiendan que se difundan públicamente el conflicto de intereses y una explicación de las partes involucradas sobre la forma en que lo están manejando.

El manejo de los conflictos de intereses es particularmente importante respecto a los auditores externos, cuya independencia es crucial para la integridad del mercado financiero. Los Principios apoyan los Principios de Supervisión del Auditor, emitidos en 2002 por la Organización Internacional de las Comisiones de Valores (IOSCO, por las siglas en inglés de International Organization of Securities Commissions), en los cuales se recomienda la creación de un organismo que, actuando en interés del público, se encargue de vigilar la calidad e instrumentación de las auditorías. Los Principios de la OCDE reconocen la importancia de las medidas que recientemente han tomado varios países para enfrentar la estructura sesgada de incentivos que surge cuando el auditor externo presta también otros servicios diferentes o cuando puede estar involucrado en la auditoría a su propio trabajo. El manejo de la relación con el auditor externo y el asegurar que la auditoría independiente sea de alta calidad se identifican en los Principios como uno de los deberes clave del consejo de administración.

Un marco para un gobierno corporativo eficaz necesita apoyarse en mecanismos que aseguren la integridad de quienes proporcionan información o consejos que pueden influir en las decisiones del inversionista, como son los analistas financieros, los corredores de valores y las agencias calificadoras. Esto es necesario debido a que las relaciones cercanas entre los proveedores de esos servicios y las empresas clientes abren la posibilidad de conflicto de intereses.

Para el mercado es importante saber si la empresa está siendo administrada debidamente respecto a los intereses de todos sus inversionistas. Con este fin, los Principios establecen que es esencial que la empresa revele completamente todas sus transacciones con las partes relacionadas. Estas transacciones se dan típicamente entre la empresa y las entidades en las que ella o su administración 
tienen un interés, o con accionistas importantes que incluyen sus parientes cercanos y asociados. Los Principios propugnan por que el beneficiario de una transacción de esa naturaleza esté obligado a informar al consejo de administración, el que a su vez debe revelarlo al mercado. Sin embargo, esta medida no exime a la empresa de mantener la vigilancia correspondiente.

Los Principios enfatizan la necesidad de proteger a los accionistas minoritarios, sobre todo cuando hay accionistas que controlan la empresa y cuyos intereses pueden diferir de los intereses de los otros accionistas. Lo anterior es una fuente de preocupación principalmente en jurisdicciones en las cuales es débil el marco legal y regulatorio para proteger los intereses minoritarios. Los Principios reafirman que es razonable que los inversionistas esperen que se prohiban los abusos por parte de quienes tienen el poder de la información interna de la empresa, incluyendo los accionistas que la controlan. También exhortan a los creadores de políticas a que consideren medidas para cerrar las brechas legales en los casos en los cuales no están prohibidos tales abusos de manera específica en la legislación o cuando no son eficaces las medidas para obligar al cumplimiento de la ley.

\section{¿Cómo ayudan los Principios a fortalecer la vigilancia de la empresa me- diante los consejos de administración?}

El consejo de administración sirve como el fulcro que equilibra los derechos de propiedad que gozan los accionistas con la discrecionalidad garantizada a los administradores. El buen gobierno corporativo requiere que el consejo de administración, cualquiera que sea su estructura, se enfoque en asuntos de largo plazo, tales como la evaluación de la estrategia corporativa y las actividades que pueden involucrar cambios en la naturaleza y dirección de la empresa, en lugar de tomar responsabilidades en las operaciones cotidianas. No obstante, tanto en lo colectivo como en lo individual, los miembros del consejo de administración deben tener incentivos y deberes claramente definidos para asegurar que cumpla sus funciones en forma eficaz.

Los Principios especifican responsabilidades claramente definidas para el consejo de administración que incluyen: el establecer un código de ética corporativa, asegurar el cumplimiento de las leyes y normas y supervisar los sistemas de control interno relacionados con los reportes financieros. Dicho consejo también debería ser responsable de la formulación y revelación de una política de remunera- 
ción en la cual se resalte el vínculo entre ésta y el desempeño de los ejecutivos clave y de sus miembros. En muchos países se considera ahora como una mejor práctica la creación de un comité de remuneraciones con directores independientes.

Dado que el consejo de administración y sus miembros tienen un deber fiduciario ante la compañía y todos sus accionistas, los Principios adoptan una concepción general de la independencia y objetividad del consejo de administración, en lugar de referirse simplemente a la independencia de la administración. Los consejos de administración deben revisar las transacciones con partes relacionadas valiéndose de miembros del consejo independientes y proporcionar acceso confidencial a informantes que puedan estar en posición de identificar conductas no éticas y transacciones abusivas. A pesar de que en los últimos años se ha propagado la práctica de crear comités del consejo de administración para tareas tales como auditoría, remuneración y nominación de miembros del consejo, no siempre se han entendido bien los conceptos subyacentes y a menudo los comités desempeñan papeles muy diferentes dependiendo de la empresa. Para evitar la confusión y para mantener informados a los inversionistas, los Principios aconsejan que la composición, el mandato y disolución de los comités se definan de forma clara y se revelen totalmente.

\section{¿Cómo pueden utilizar los Principios de la OCDE los gobiernos?}

Aunque varias de las figuras aconsejadas por los Principios requieren acciones por parte de los consejos de administración y de los inversionistas, entre otros, también las autoridades gubernamentales deben jugar un papel importante. Por ejemplo, usualmente los consejos de administración deberán ser capaces de adoptar una estructura congruente con una supervisión eficaz y con la rendición de cuentas a los accionistas. Sin embargo, figuras deseables como la cooperación entre los inversionistas y la protección de los accionistas minoritarios pueden depender en gran medida de las acciones emprendidas por el gobierno para eliminar barreras regulatorias y reforzar sus derechos. Además, las acciones del sector privado por sí solas podrían no conducir a las prácticas de gobierno corporativo deseables. Por ejemplo, cuando la administración se encuentra atrincherada y los mercados de capitales son débiles, los consejos de administración pueden continuar evadiendo sus responsabilidades a menos que las autoridades emprendan acciones para remediarlo. 
Los Principios ofrecen una guía amplia que los gobiernos pueden seguir para revisar si su marco para el gobierno corporativo es compatible con el gobierno corporativo que desean. Se anima a los creadores de políticas a que desarrollen el marco del gobierno corporativo sin perder de vista su impacto en el desempeño de la economía general, en la integridad del mercado y los incentivos que ésta crea para los participantes del mercado, así como en la promoción de la transparencia y de mercados emergentes. Esto debe ayudar tanto a reducir el riesgo de un costoso exceso de regulación como a disminuir al mínimo las consecuencias no intencionales de las medidas políticas. Para sostener la integridad del mercado, los requisitos legales y regulatorios relacionados con las prácticas del gobierno corporativo deben ser congruentes con el imperio de la ley, la transparencia y la coactividad.

Los Principios también abarcan los tipos de mecanismos que las partes deben establecer para proteger sus derechos. Las autoridades de supervisión, regulación y las encargadas de obligar al cumplimiento de la ley deben tener la autoridad, la integridad y los recursos para cumplir cabalmente sus deberes de una forma profesional y objetiva. No obstante, si los recursos institucionales están restringidos, deben adecuarse los requerimientos legales y regulatorios. Por ejemplo, cuando los tribunales son débiles, se podría dar mayor peso a otros mecanismos para proteger los derechos de los accionistas, tales como la reducción del porcentaje accionario requerido para poder convocar a asambleas de accionistas y proponer miembros del consejo de administración, o elevar los márgenes de votación requeridos para decisiones de importancia. Los fallos judiciales deben ser explicados oportunamente, en forma transparente y de manera completa. El cuadro 2 expone en detalle la ayuda que la OCDE ofrece a los gobiernos para que éstos mejoren el gobierno corporativo. 


\section{Cuadro 2. Actividades de la OCDE para mejorar el gobierno corporativo}

A través de su Grupo de Conducción sobre el Gobierno Corporativo y las Mesas Redondas Regionales sobre Gobierno Corporativo, la OCDE sirve como el nexo internacional para las discusiones de políticas relacionadas con el gobierno corporativo. Los Principios son la pieza central de las diversas actividades que ha emprendido este organismo para mejorar el gobierno corporativo. En cooperación con el Banco Mundial, las Mesas Redondas Regionales han utilizado dichos principios como marco del diálogo sobre las políticas para promover las reformas regionales del gobierno corporativo en Asia, América Latina, Eurasia, el Sudeste Europeo y Rusia. Como resultado, se han obtenido los documentos (White Papers) en los que se desarrollan objetivos comunes para las políticas y se resaltan las recomendaciones para las acciones políticas. El conocimiento ganado a partir de las mesas redondas se ha puesto a disposición del público y se resume en Experiences from the Regional Corporate Governance Roundtables, reporte de la OCDE en que se comparan los problemas de gobierno corporativo que enfrentan muy diferentes economías con mercados emergentes y en vías de desarrollo y en el que se abordan detalladamente las prioridades que han establecido esas regiones. A su vez, esta experiencia influyó en la revisión de los Principios.

El buen gobierno corporativo también es necesario para las empresas estatales. La función del Estado como propietario en las empresas de las que es accionista se encuentra lejos de estar totalmente resuelta, incluso aún después de considerar los efectos benéficos de la privatización parcial que en muchos países ha abierto el camino a iniciativas de reestructuración sin precedentes y ha incrementado la exposición a la competencia con las entidades privadas. El Grupo de Trabajo de la OCDE sobre la Privatización y el Gobierno Corporativo de la Empresas Estatales está desarrollando un conjunto de pautas que se terminarán en 2005 y que permitiran que los países cuenten con mejores puntos de referencia para las funciones del Estado como propietario. Este trabajo está sujeto a un proceso de consulta abierta.

A menudo se ha asociado la reforma regulatoria con cambios en el marco del gobierno corporativo. La respuesta de la OCDE a la demanda creciente de información actualizada, completa y comparable sobre los desarrollos regulatorios recientes ha sido la creación de la bases de datos sobre Derecho y Gobierno Corporativos, una herramienta interactiva única para la diseminación de la información legal y regulatoria relacionada con el gobierno corporativo. 


\section{¿Cómo se efectuó la revisión de los Principios?}

Como resultado de una serie de escándalos corporativos que habían socavado la confianza en la integridad tanto de las empresas como de las instituciones y de los mercados financieros, en 2002 los ministros de la OCDE convocaron a que en 2004, un año antes de lo anteriormente establecido, se llevase a cabo una evaluación de los Principios de la OCDE. Para apoyar su trabajo, los ministros solicitaron una encuesta sobre los desarrollos relacionados con el gobierno corporativo con el objetivo de identificar lo que podría aprenderse y sus posibles implicaciones para los Principios.

La evaluación se condujo bajo la responsabilidad del Grupo de Conducción nombrado por la OCDE y con la participación activa de observadores de instituciones internacionales clave, destacando entre ellos: el Banco de Pagos Internacionales, el Fondo Monetario Internacional, el Banco Mundial, el Foro de Estabilidad Financiera, la Organización Internacional de Comisiones de Valores y el Comité de Basilea. Sobre una base ad hoc, también participaron en las reuniones representantes de empresas líderes y de trabajadores, como el Comité Consultivo de la OCDE sobre Empresa e Industria y el Comité Consultivo de la OCDE sobre Organizaciones Sindicales.

El Grupo de Conducción envió un cuestionario a los países miembros solicitando información acerca de los asuntos relacionados con el gobierno corporativo, las fuerzas actuantes y las políticas propuestas. Junto con una revisión de las prácticas en los países miembros, las respuestas formaron la base del reporte titulado Corporate Governance: A Survey of Developments in OECD Countries, y sirvieron como información para las discusiones del Grupo de Conducción.

Al llevar a cabo su revisión, el Grupo de Conducción organizó consultas amplias y transparentes, incluyendo en el proceso de evaluación las consultas con amplios estratos de los sectores privado, laboral y de la sociedad civil. Conjuntamente con las reuniones del Grupo de Conducción, hubo tres grandes reuniones de consulta con una amplia participación. Adicionalmente, la Secretaría General de la OCDE convocó a dos mesas redondas informales con representantes importantes de las organizaciones internacionales clave, las empresas y de los trabajadores.

Las consultas con los socios no miembros se llevaron a cabo primero por medio de reuniones de las cinco Mesas Redondas Regionales sobre Gobierno Corporativo, obteniéndose resultados adicionales en una reunión especial que se organizó con la 
colaboración del Foro Global sobre Gobierno Corporativo y a la que asistieron 43 países no miembros.

En enero de 2004 se colocó en internet un borrador de los Principios revisados para recabar comentarios del público en general, recibiéndose aproximadamente 75 de individuos, asociaciones profesionales, empresas y sindicatos de trabajadores. Cuando se obtuvo autorización, esos comentarios se colocaron en el sitio web de la OCDE para que el público tuviese acceso.

\section{¿Qué sigue?}

Los Principios deben considerarse un documento vivo. Una prioridad de la OCDE es asegurarse de que se difundan ampliamente y de que se practiquen. Esto incluye un diálogo continuo en el cual los creadores de políticas, reguladores y quienes fijan la normatividad puedan intercambiar experiencias prácticas de la instrumentación de los principios. La OCDE también continuará dando seguimiento a los desarrollos e identificando las nuevas tendencias y desafíos que merezcan atención. Como parte importante del trabajo futuro, la OCDE auspiciará el diálogo internacional entre los múltiples grupos de interesados en el gobierno corporativo. Este diálogo entre las empresas, los inversionistas, los proveedores de servicios, los trabajadores y otros será tan inclusivo como sea posible y proporcionará una oportunidad importante para asegurar que los principios de la OCDE sigan siendo pertinentes y se utilicen en el sector privado. Más allá de los principios, las pautas para el gobierno corporativo de las empresas del Estado se volverán un aspecto de dicho diálogo una vez que sean acordadas.

Ya está en marcha la siguiente fase del proceso de las mesas redondas regionales para los países que no son miembros de la OCDE. En el caso de la mesa redonda rusa los participantes se han puesto de acuerdo para crear dos grupos de trabajo ad hoc para examinar las opciones de políticas en dos áreas prioritarias: la transición hacia las normas de reportes financieros reconocidas internacionalmente y los problemas que surgen tanto de las transacciones entre partes relacionadas como de la transparencia en condiciones de concentración de la propiedad y el control. Las mesas redondas de Asia, América Latina y el Sudeste Europeo centrarán su atención en la instrumentación y la puesta en marcha de las recomendaciones establecidas en los documentos de la OCDE. Dentro de poco tiempo la mesa redonda de Eurasia publicará una visión general comparativa del gobierno corporativo en la región, que incluye las prioridades de las acciones adicionales que deben efectuarse en la fase de seguimiento. 